\title{
ANTI-VEGF THERAPY IN CENTRAL RETINAL VEIN OCCLUSIONS
}

\author{
Meidina Rahmah, Ramzi Amin, AK Ansyori \\ Department of Ophthalmology, Universitas Sriwijaya
}

\begin{abstract}
Introduction: Central retinal vein occlusion (CRVO) is the second most common retinal vascular disease after diabetic retinopathy and is estimated to affect more than 16 million adults worldwide. Treating the underlying cause of occlusion of the central retinal vein, reducing the risk factors, and early and proper management are the keys toward a better prognosis in patients with CRVO. Repeated frequent treatment of CRVO with macular edema with intravitreal anti-vascular endothelial growth factor (anti-VEGF) is an effective treatment to improve visual outcome. In this case report we assess the 3-month efficacy of antiVEGF therapy in CRVO.
\end{abstract}

Method: In this case, a 52-year-old man presented with an acute loss of vision over the last week in his left eye. His baseline visual acuity was 1/60 and his central retinal thickness (CRT) on Optical Coherence Tomography (OCT) was $523 \mu \mathrm{m}$. Fundus examination revealed a hemorrhage in all four quadrants, hard exudates, swelling of the optic nerve, and macular edema. Laboratory test confirms hyperlipidemia and we consult this patient to Internal Medicine Department. This patient got 3 monthly injections of 1,25 mg Bevacizumab.

Results: By 3 months, the OCT appeared fairly normal and the visual acuity improved to 6/60

Conclusion: Anti-VEGF therapy is effective for treating macular edema secondary to CRVO

Keywords: central retinal vein occlusion, anti-vascular endothelial growth factor, bevacizumab, macular edema

Cite This Article: RAHMAH, Meidina. Anti-VEGF Therapy In Central Retinal Vein Occlusions. International Journal of Retina, [S.I.], v. 1, n. 2, aug. 2018. ISSN 2614-8536. Available at: <https://www.ijretina.com/index.php/ijretina/article/view/42>.

*Correspondence to: Meidina Rahmah, Department of Ophthalmology, Universitas Sriwijaya, meidinarahmah23@gmail.com

\section{INTRODUCTION}

Central Retinal Vein Occlusion (CRVO) is a retinal vascular disease caused by acute obstruction of central retinal vein characterized by abrupt onset visual loss. CRVO is found to be the second most common retinal vascular disease following diabetic retinopathy and is estimated to affect more than 16 million adults worldwide. $^{1-5}$ Increasing age and existing cardiovascular disorders, including hypertension, hyperlipidemia, and diabetes mellitus are the most common systemic risk factors leading to CRVO. ${ }^{1-3}$

The pathogenesis of CRVO is the blockage of venous circulation causes an elevation of intraluminal pressure in the capillaries, leading to hemorrhages and leakage of fluid within the retina, increase of interstitial pressure and a consequent reduction of retinal perfusion. When blood flow is disrupted, adequate levels of oxygen fail to reach the retina, triggering the release of vascular endothelial growth factor (VEGF). VEGF increases the permeability of blood vessels, leading to swelling in the central part of the retina-a condition known as macular edema, the leading cause of vision loss from CRVO. ${ }^{1-3}$

Management of CRVO is complex, and in general, it aims to improve perfusion, treat the underlying etiologies and prevent further complications. Various complications will result from poorly treated CRVO, including neovascularization, vitreous hemorrhage, and neovascular glaucoma. ${ }^{1-5}$ Cugati et al reported that $68-72 \%$ patients with CRVO that left untreated had increased intraocular pressure due to neovascular glaucoma. Neovascularization of iris can occur within 6 months post occlusion in untreated cases of CRVO. ${ }^{1,3,6}$ 


\section{METHOD}

A 52-year old man came with the chief complaint of having abrupt onset of blurry vision with no history of red or painful eye and curtain-covered vision. From the ophthalmology examination, it was noted that the visual acuity was $1 / 60$ which could not be corrected. On clinical examination, there were no signs of neovascularization of the iris. From funduscopy examination, there were dot, blot, and flame-shaped haemorrhage in all quadrants of retina, exudate in all quadrants, tortuous vein, decreased foveal reflex and hyperemia of the optic disc with poorlydefined border (fig 1). OCT finding showed central retinal thickness (CRT) of $523 \mu \mathrm{m}$ (fig 2).

According to the physical and laboratory examination the patient suffered hypertension with high total cholesterol level (224 mg/dL), low HDL level (48 mg/dL), high LDL level (176 mg/dL), and low triglyceride level (116 $\mathrm{mg} / \mathrm{dL})$.

\section{RESULTS}

The patient was treated with anti-VEGF therapy with 1.25 mg Bevacizumab administered as intravitreal injection. Follow-up was done right after anti-VEGF administration and from then on, there was improvement on both visual acuity and macular oedema. At 1 month post anti-VEGF injection, further improvement of visual acuity was noted as it reached $4 / 60$, and we conducted OCT examination accordingly and it was found that the macular oedema still persisted with central retinal thickness (CRT) was $314 \mu \mathrm{m}$, therefore the patientreceived the second injection of Bevacizumab. The patient was evaluated again at 1 month and it was found that the visual acuity was better and the macular oedema was slightly reduced. Accordingly, the patient got the third Bevacizumab injection and evaluation at 1 month post the third injection showed no macular oedema. Unfortunately, we do not have fundus photograph and OCT after 3 months treatment.

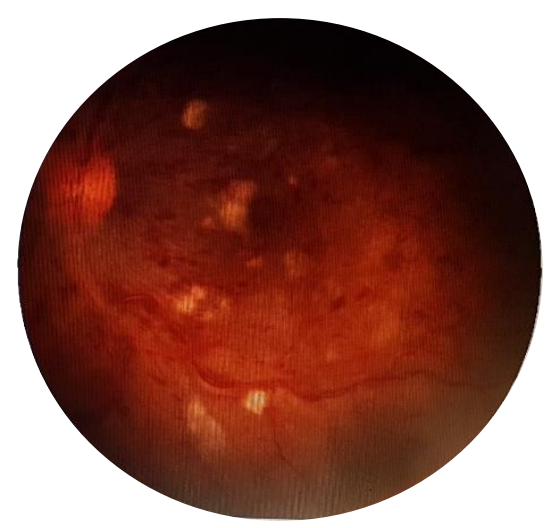

Figure 1. Fundus photograph before first intravitreal anti-VEGF

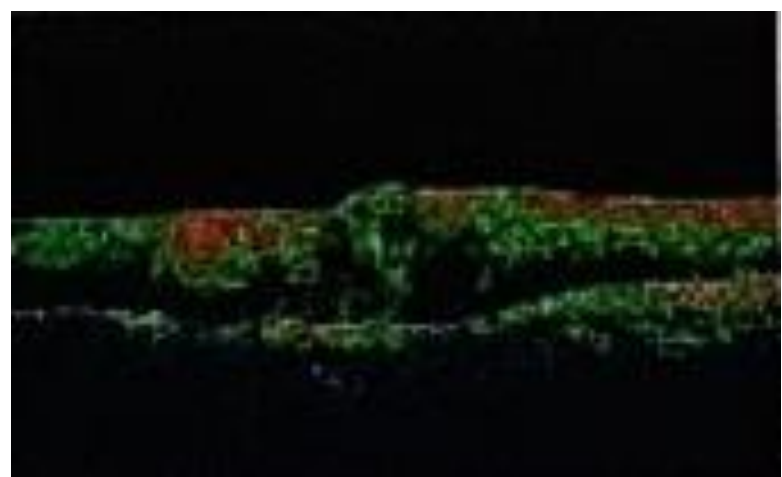

Figure 2. Optical Coherence Tomography (OCT) before first intravitreal anti-VEGF

\section{DISCUSSION}

A decrease in visual acuity in CRVO is caused by macular oedema. VEGF is considered as the main actor causing macular oedema in RVO. Intravitreal anti-VEGF is useful for reducing macular oedema, therefore improvement in visual acuity can be achieved. The faster the macular oedema is treated, the better the response to therapy and the prognosis of a patient with CRVO. ${ }^{5}$

The patient in this report was given anti-VEGF therapy with intravitreal injection of $1.25 \mathrm{mg}$ Bevacizumab. 70
Bevacizumab acts through inhibition of VEGF-A from binding to its receptor on the surface of vascular endothelial cells resulting in reduction of proliferation of endothelial cells, vascular leakage, and formation of neovascularization. The patient got a total of three injection of Bevacizumab administered every month giving to improvement of visual acuity and reduction of macular oedema.

According to BRIGHTER study, after intravitreal injection of anti-VEGF given in three consecutive months, the Published by : INAVRS https://www.inavrs.org/ | International Journal of Retina https://ijretina.com 2018; 1; 2; 
patient should be reevaluated for improvement of visual acuity and OCT examination should also be conducted. If the result from the OCT showed persistent fluid, despite of improved or stable visual acuity, it is recommended to give the patient the second dose of anti-VEGF. If there is no reduction of macular oedema and visual acuity is not improved or even getting worse, it is recommended to conduct fluorescence angiography to assess for any possibilities of ischemic lesion in the maculae of peripheral part of retina. Patients with normal OCT result should be routinely evaluated and treated using pro re nata (PRN) approach. Intravitreal steroid injection or antiVEGF replacement therapy can be given to patients who do not respond to anti-VEGF therapy. ${ }^{7}$

If there is neovascularization that potentially cause vitreous hemorrhage, photocoagulation laser directed to the ischemic area of the peripheral retina is the treatment of choice. Additionally, anti-VEGF can also be applied, however, monotherapy with anti-VEGF is not the first choice in the management of neovascularization following retinal vein occlusion. Central Vein Occlusion Study (CVOS) concluded that panretinal photocoagulation laser has no benefit if conducted before neovascularization is formed. ${ }^{5}$

In addition to intravitreal anti-VEGF, the patient can also be given intravitreal steroid injection. SCORE-CRVO study showed improvement of visual acuity at 12 months post intravitreal triamcinolone injection. Unfortunately, there was also high incidence of cataract in this study population. Hence, intravitreal steroid injection is not recommended. ${ }^{8}$

\section{REFERENCES}

1. Cugati S, Wang JJ. Retinal vein occlusion and vascular mortality: pooled data Analysis of 2 populationbased cohorts. Ophthalmology. 2007. 114: 520-524

2. American Academy of Ophthalmology. Retina and vitreous. In Basic and Clinical Science Course, Section 12. 2014-2015

3. Ryan, stephen J. Retina volume1-2. Mosby Company. Baltimore. 2010

4. Kanski, Jack. Clinical Ophthalmology A Systematic Approach. Seventh edition. 2011. Elsevier

5. Lattanzio R. Torres G, et al. Retinal Vein Occlusion: Current Treatment. Ophthalmologica. 2011. 225:135143

6. The royal college of ophthalmologist. Retinal vein occlusion guidelines. United Kingdom. London. 2015

7. Pielen, A., et al. Efficacy and safety of intravitreal therapy in macular edema due to branch and central retinal vein occlusion: a systematic review. PLoS One 2013. 8(10)

8. Gewaily D, Greenberg PB. Intravitreal steroids versus observation for macular edema secondary to central retinal vein occlusion. Cochrane Database Syst. 2009.

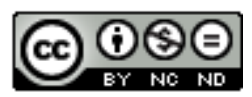

This work licensed under Creative Commons Attribution 